\title{
Monte Carlo simulations of Higgs-boson production at the LHC with the KrkNLO method
}

\author{
S. Jadach ${ }^{1}$, G. Nail ${ }^{2,3}$, W. Płaczek ${ }^{4}$, S. Sapeta ${ }^{1,5}$, A. Siódmok ${ }^{1,5, a}$, M. Skrzypek ${ }^{1}$ \\ ${ }^{1}$ Institute of Nuclear Physics, Polish Academy of Sciences, ul. Radzikowskiego 152, 31-342 Kraków, Poland \\ 2 Particle Physics Group, School of Physics and Astronomy, University of Manchester, Oxford Road, Manchester M13 9PL, UK \\ ${ }^{3}$ Institute for Theoretical Physics, Karlsruhe Institute of Technology, Wolfgang-Gaede-Strasse 1, 76131 Karlsruhe, Germany \\ ${ }^{4}$ Marian Smoluchowski Institute of Physics, Jagiellonian University, ul. Łojasiewicza 11, 30-348 Kraków, Poland \\ 5 Theoretical Physics Department, CERN, Geneva 23 1211, Switzerland
}

Received: 24 October 2016 / Accepted: 3 March 2017 / Published online: 16 March 2017

(C) The Author(s) 2017. This article is published with open access at Springerlink.com

\begin{abstract}
We present numerical tests and predictions of the KrkNLO method for matching of NLO QCD corrections to hard processes with LO parton-shower Monte Carlo generators (NLO+PS). This method was described in detail in our previous publications, where it was also compared with other NLO+PS matching approaches (MC@NLO and POWHEG) as well as fixed-order NLO and NNLO calculations. Here we concentrate on presenting some numerical results (cross sections and distributions) for $Z / \gamma^{*}$ (DrellYan) and Higgs-boson production processes at the LHC. The Drell-Yan process is used mainly to validate the KrkNLO implementation in the Herwig 7 program with respect to the previous implementation in Sherpa. We also show predictions for this process with the new, complete, MC-scheme parton distribution functions and compare them with our previously published results. Then we present the first results of the KrkNLO method for Higgs production in gluon-gluon fusion at the LHC and compare them with MC@NLO and POWHEG predictions from Herwig 7, fixed-order results from HNNLO and a resummed calculation from $\mathrm{HqT}$, as well as with experimental data from the ATLAS collaboration.
\end{abstract}

\section{Introduction}

The discovery of the Higgs boson, at the Large Hadron Collider (LHC) [1,2], opened a new era in the exploration of the electroweak sector of the standard model (SM). The measured value of the Higgs mass uniquely specifies all of the couplings and turns the SM into a fully predictive theory.

This work is partly supported by the Polish National Science Centre Grant UMO-2012/04/M/ST2/00240.

\footnotetext{
a e-mail: andrzej.siodmok@cern.ch
}

Hence, we are at a position to perform stringent tests of our current modelling of these fundamental interactions. This is only possible if we are in possession of precise theoretical predictions for the Higgs-production cross sections.

Most of the Higgs-boson particles observed at hadron colliders are produced through the process of gluon fusion, a channel that is known to exhibit very slow convergence in perturbative quantum chromodynamics (QCD). At LHC energies, the next-to-leading order (NLO) corrections to the total cross section for the inclusive production of the Higgs boson through gluon fusion turn out to be as large as $70 \%$, and the next-to-next-to-leading order (NNLO) corrections increase the cross section by another $30 \%$ [3-5]. The theoretical uncertainty of the NNLO result, arising from the missing higher orders and obtained by the standard renormalization and factorization scale variations, is estimated at around $10 \%$, and is hence at the level of the experimental accuracy of the Run 1 LHC measurements. This large uncertainty at NNLO has motivated the efforts to further improve the precision by calculating the full next-to-next-to-next-to-leading order $\left(\mathrm{N}^{3} \mathrm{LO}\right)$ result for inclusive Higgs-boson production in gluon fusion [6]. Adding these contributions to the predictions for the cross section reduces their scale uncertainties down to the level of $3 \%$.

Apart from the inclusive Higgs cross section, which is the most fundamental quantity, as it enables one to predict the total number of Higgs particles produced at a given energy and luminosity, one is also equally interested in more differential observables. Therefore, a significant amount of work has also gone into obtaining predictions for differential cross sections for Higgs production in gluon fusion beyond NLO.

In particular, differential observables have been predicted within frameworks of analytic resummation, like for example small- $q_{T}$ resummation performed in QCD in coordinate 
space up to the NNLL+NLO accuracy [7] and directly in momentum space up to NNLL+NNLO [8] as well as within SCET [9] up to NNLL+NLO.

Differential cross sections for Higgs production in gluon fusion have also been widely studied with approaches in which fixed-order NLO results are matched to parton shower (NLO+PS) such as the MiNLO method [10, 11]. Recently, NNLO+PS matched results were computed with the $\mathrm{UN}^{2}$ LOPS technique [12] as well as with an extended version of MiNLO [13-15] combined with the HNNLO program $[5,16]$. The current methods of performing NNLO+PS [11,14,15,17-21] represent clear progress in the matching of fixed-order NNLO QCD calculations with parton-shower Monte Carlos (PSMCs). The next challenge towards even higher-precision perturbative QCD calculations, needed until the end of the LHC era two decades from now, is the combination of the fully exclusive NNLO corrections for the hard process with NLO parton shower (NNLO+NLOPS).

In this article, we present NLO+PS predictions for various differential distributions computed with the KrkNLO approach [22,23]. The main advantage of the KrkNLO method with respect to other methods of matching the fixed-order NLO calculations with PSMCs (MC@NLO and POWHEG) is its simplicity, which stems from the fact that the entire NLO corrections are implemented using a simple, positive, multiplicative MC weight in combination with pre-calculated MC-scheme PDFs. The present work is relevant for the above future developments in the sense that it presents a simplified method of correcting the hard process to NLO level in combination with a leading-order (LO) parton shower (PS) that will hopefully pave the way to NNLO hard processes combined with a NLO PSMC; NLOPS is a parton-shower MC implementing the NLO evolution kernels in the fully exclusive form, thus providing the full set of the soft-collinear counter-terms for the hard process. Reference [24] reviews several feasibility studies which show that constructing such a NLOPS is, in principle, plausible. In our opinion, any simplifications of the NLO+PS matching, as in the KrkNLO method, will be instrumental in the progression towards more ambitious fully exclusive NNLO+NLOPS projects.

The KrkNLO method was first introduced Ref. [25] for $Z / \gamma^{*}$ production in hadron collisions (the Drell-Yan process, DY) and was also presented in Ref. [23]. These developments followed the initial study in Refs. $[22,26]$ on the inclusion of fixed-order NLO QCD corrections to the hard process in LO PSMC through an appropriate Monte Carlo weight. This study was performed with the use of some dedicated toy-model parton-shower generator and for gluonstrahlung from quarks only, albeit for two processes: DY production and deep-inelastic electron-proton scattering (DIS).
The first realistic numerical results (total cross sections and distributions of $Z / \gamma^{*}$ transverse momentum and rapidity) of the KrkNLO method, based on its implementation in the Sherpa [27] PSMC, were presented in Ref. [25] for the DY process. The KrkNLO results were compared with the fixed-order NLO predictions of MCFM [28] and those of other NLO-PSMC matching methods, namely MC@NLO [29,30] and POWHEG [31,32], as well as with the fixed-order NNLO calculations of DYNNLO [33]. A satisfactory agreement with other NLO calculations was found. Moreover, for the boson transverse momentum the agreement with the NNLO predictions was better than for MC@NLO and POWHEG, which may be explained by effects beyond NLO accounted for in KrkNLO as a result of using the MC factorization scheme and multiplicative virtual+soft-real corrections; see Ref. [25] for more details. In that paper the advantages of the KrkNLO method over the MC@NLO and POWHEG techniques were also discussed.

In Ref. [25] the concept of the Monte Carlo (MC) factorization scheme was introduced as a necessary ingredient of the KrkNLO method; this was further developed in Ref. [34]; see also Ref. [35]. Appropriate MC-scheme parton distribution functions (PDFs) were defined and constructed from PDFs in the standard $\overline{\mathrm{MS}}$ scheme. However, those MC-scheme PDFs included only contributions from parton-parton transitions that were sufficient for the DY process in the NLO approximation-in the following we denote them with $\mathrm{MC}_{\mathrm{DY}}$. The complete PDFs in the MC factorization scheme, which include all of the LO parton-parton transitions, were defined, computed and compared with the $\overline{\mathrm{MS}}$ PDFs in Ref. [34], where Higgs-boson production from initial-state gluons was considered. In that paper only the values of the total cross section for the Higgs production at the LHC were shown; further results for this process will be presented in this work. However, before presenting the Higgs results, we first validate the implementation of the KrkNLO method in the Herwig 7 program with respect to its previous implementation in Sherpa using the DY process. The Herwig 7 implementation of the KrkNLO method will be our basic platform for its future developments and applications to other processes. Then we will compare the KrkNLO results for the DY process based on the complete MC-scheme PDFs with those where the $\mathrm{MC}_{\mathrm{DY}} \mathrm{PDFs}$, as defined in Ref. [25], are used.

For the process of Higgs production in gluon-gluon fusion, we present the results for the total cross section and the distributions of the Higgs transverse momentum and rapidity at the LHC. The predictions of the KrkNLO method are compared with those of the fixed-order NLO and NNLO calculations of HNNLO [5], the results of the NLO-PSMC matching approaches of MC@NLO [30] and POWHEG [31,32], as well as resummed calculations from $\mathrm{HqT}[7,36]$. We also 
confront the predictions of all the above matching methods with the LHC data of the ATLAS experiment [37].

The outline of this paper is the following: In Sect. 2, after describing the set-up for our numerical computations, we present and discuss the results of the KrkNLO method; we do this first for the DY process and then for Higgs production in gluon-gluon fusion at the LHC. Section 3 concludes our work and provides some outlook for future developments. In Appendix A we present comparisons of various PDF parametrizations in the $\overline{\mathrm{MS}}$ and MC factorization schemes.

\section{Numerical results}

\subsection{Set-up}

For the numerical evaluation of the cross sections ${ }^{1}$ at the LHC for the proton-proton collision energy of $\sqrt{s}=8 \mathrm{TeV}$ we have chosen the following set of the Standard Model input parameters:

$$
\begin{array}{rlrl}
M_{Z} & =91.1876 \mathrm{GeV}, & \Gamma_{Z} & =2.4952 \mathrm{GeV}, \\
M_{W} & =80.4030 \mathrm{GeV}, & \Gamma_{W} & =2.1240 \mathrm{GeV}, \\
M_{H} & =126 \mathrm{GeV}, & m_{t} & =173.2 \mathrm{GeV}, \\
& G_{\mu} & =1.16637 \times 10^{-5} \mathrm{GeV}^{-2},
\end{array}
$$

and use the $G_{\mu}$ scheme [38] for the electroweak sector of the Standard Model. We take the renormalization and factorization scales to be $\mu_{R}^{2}=\mu_{F}^{2}=M_{B}^{2}$, where $M_{B}$ is the mass of the $Z$ or Higgs boson for the respective processes. In the case of the Drell-Yan process, detector acceptance cuts are imposed only on the invariant mass of the final-state lepton pair $\left(Z / \gamma^{*}\right.$-boson):

$$
50 \mathrm{GeV}<M_{\bar{l}}<150 \mathrm{GeV} .
$$

For the Higgs-production process we do not apply any cuts, and for simplicity we set the Higgs boson to be stable. The LO, NLO and NNLO Higgs-production matrix elements are calculated in the $m_{t} \rightarrow \infty$ and $m_{q \neq t}=0$ approximation. To compute the hadronic cross section, we employ $\overline{\mathrm{MS}} \mathrm{PDFs}$ from the LHAPDF library [39] and their MC-scheme counterparts when using KrkNLO. The value of $\alpha_{s}$ is chosen to match the value used in the PDFs. The PDF set used is detailed in the relevant subsection.

\subsection{Drell-Yan process}

The results of the KrkNLO method implemented on top of the Sherpa PSMC [27] for the Drell-Yan process were already presented in Ref. [25]. As with these results, for this process we use the MSTW2008 LO set of parton distribution

\footnotetext{
$\overline{1}$ Unless stated otherwise in the text.
}

Table 1 Values of the total cross section with statistical errors at the Born level for the Drell-Yan process with PDFs in the $\overline{\mathrm{MS}}$ factorization scheme

\begin{tabular}{llll}
\hline & MCFM & Sherpa & Herwig 7 \\
\hline$\sigma_{\text {tot }[\mathrm{pb}]}$ & $936.9(1)$ & $937.2(2)$ & $936.6(2)$ \\
\hline
\end{tabular}

functions [40], which has $\alpha_{S}\left(M_{Z}^{2}\right)=0.13938690$. Here we use these Sherpa results to validate a new implementation of KrkNLO in the Herwig 7 program [41,42]. This version of the Herwig 7 PSMC features a new parton-shower algorithm based on the Catani-Seymour (CS) dipole [43] formalism, and is therefore well suited to the implementation of the KrkNLO method. Details on how to implement the KrkNLO method in the CS-dipole PSMC are given in Ref. [25] for the DY process and in Ref. [34] for the Higgs-boson production. In short, it amounts to replacing the $\overline{\mathrm{MS}}$ PDFs with the MC-scheme PDFs and applying to each event generated by the PSMC an appropriate Monte Carlo weight that introduces the NLO QCD correction to a given hard process. This weight is positive-definite and can be computed simply from information provided in an event record.

\subsubsection{LO results}

As a basis for this validation, we first compare the LO-level predictions from MCFM and Sherpa with those of Herwig 7 using an identical choice of parameters (see Sect. 2.1). The results for the total cross section presented in Table 1 show a very good agreement (within statistical errors) between different programs.

Since the KrkNLO method depends on details of the parton-shower algorithm ${ }^{2}$ both dipole showers $[44,45]$ have to be set as similar as possible. The level of agreement of the two PSMCs is presented in Fig. 1, where we show distributions of the final-state $e^{+} e^{-}$-pair (the decay product of $Z / \gamma^{*}$ boson) transverse momentum $p_{T e e}$, invariant mass $m_{e e}$ and rapidity $y_{e e}$. We can see that all of the distributions are in good agreement. In the case of the transverse-momentum distribution there are some differences at small $p_{T}$ that result from different treatment of intrinsic- $k_{T}$ and the soft-parton limit in the two programs, where differences in the latter emerge from the different ordering variables employed in the two programs. The differences at high $p_{T}$ are due to limited statistics in this region.

\footnotetext{
$\overline{2}$ For example, in Sherpa the initial-state parton shower has a prefactor of $1 / 2$ in the scale of the running $\alpha_{s}$ in the calculation of the Sudakov form factor.
} 

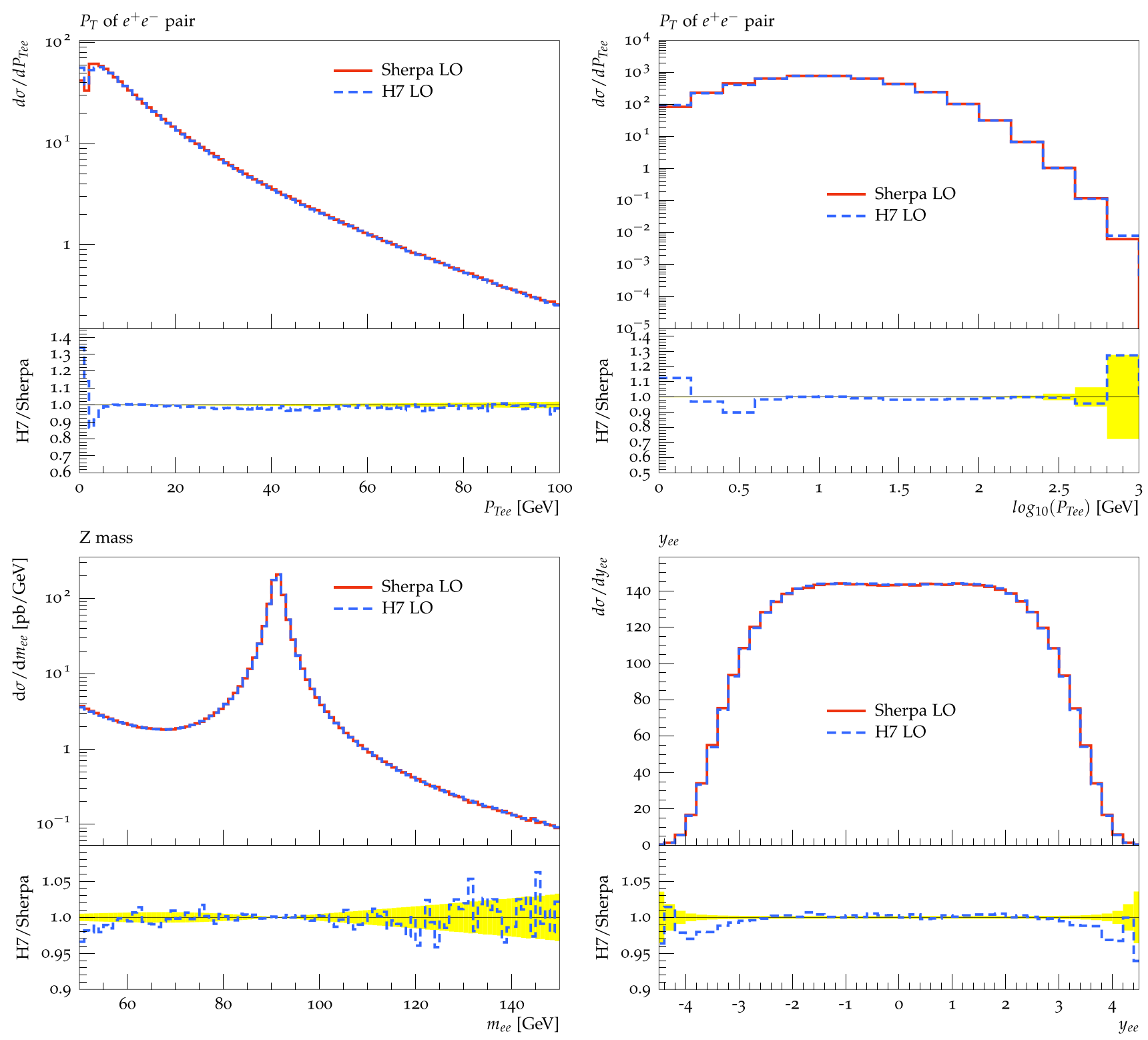

Fig. 1 Comparisons of the $Z / \gamma^{*}$ distributions from Sherpa and Herwig 7 for the LO-level Drell-Yan process with $e^{+} e^{-}$pairs in the final state

\subsubsection{KrkNLO results: $H 7$ vs. Sherpa implementations}

With the consistent predictions obtained at the LO level, we are now ready to examine the consistency between the Sherpa and Herwig 7 implementations of KrkNLO. For the comparisons, we consider both the $q \bar{q}$ and $q g$ NLO channels of the DY process, with the backward evolution of the parton shower running to the end, as in the standard PSMC set-up. In this set-up the argument of $\alpha_{s}$ in the hard-real NLO corrections is the evolution variable $q^{2}$, i.e. $\alpha_{s}\left(q^{2}\right)$, and in the virtual+soft-real correction it is set to $M_{Z}$, i.e. $\alpha_{S}\left(M_{Z}^{2}\right)$. Here both programs use the $\mathrm{MC}_{\mathrm{DY}}$ version of PDFs, those in which only the parton-parton transitions relevant to the DY process up to NLO are considered; see Ref. [25].
Table 2 Values of the total cross section with statistical errors for the Drell-Yan process from the KrkNLO method implemented in Sherpa and Herwig 7 compared to the fixed-order result of MCFM

\begin{tabular}{llll}
\hline & MCFM & KrkNLO Sherpa & KrkNLO Herwig 7 \\
\hline$\sigma_{\text {tot }}[\mathrm{pb}]$ & $1086.5(1)$ & $1045.2(3)$ & $1046.5(2)$ \\
\hline
\end{tabular}

Once again, we start from the comparison of the total cross section-the results are collected in Table 2. We see that both implementations of the KrkNLO method give cross sections that agree at the per-mille level — the tiny residual discrepancy is due to the aforementioned differences in the low- $p_{T}$ region between Herwig 7 and Sherpa which affect the KrkNLO correcting weights. These values also agree with our previous results in Ref. [25] (see Table 5 there). 

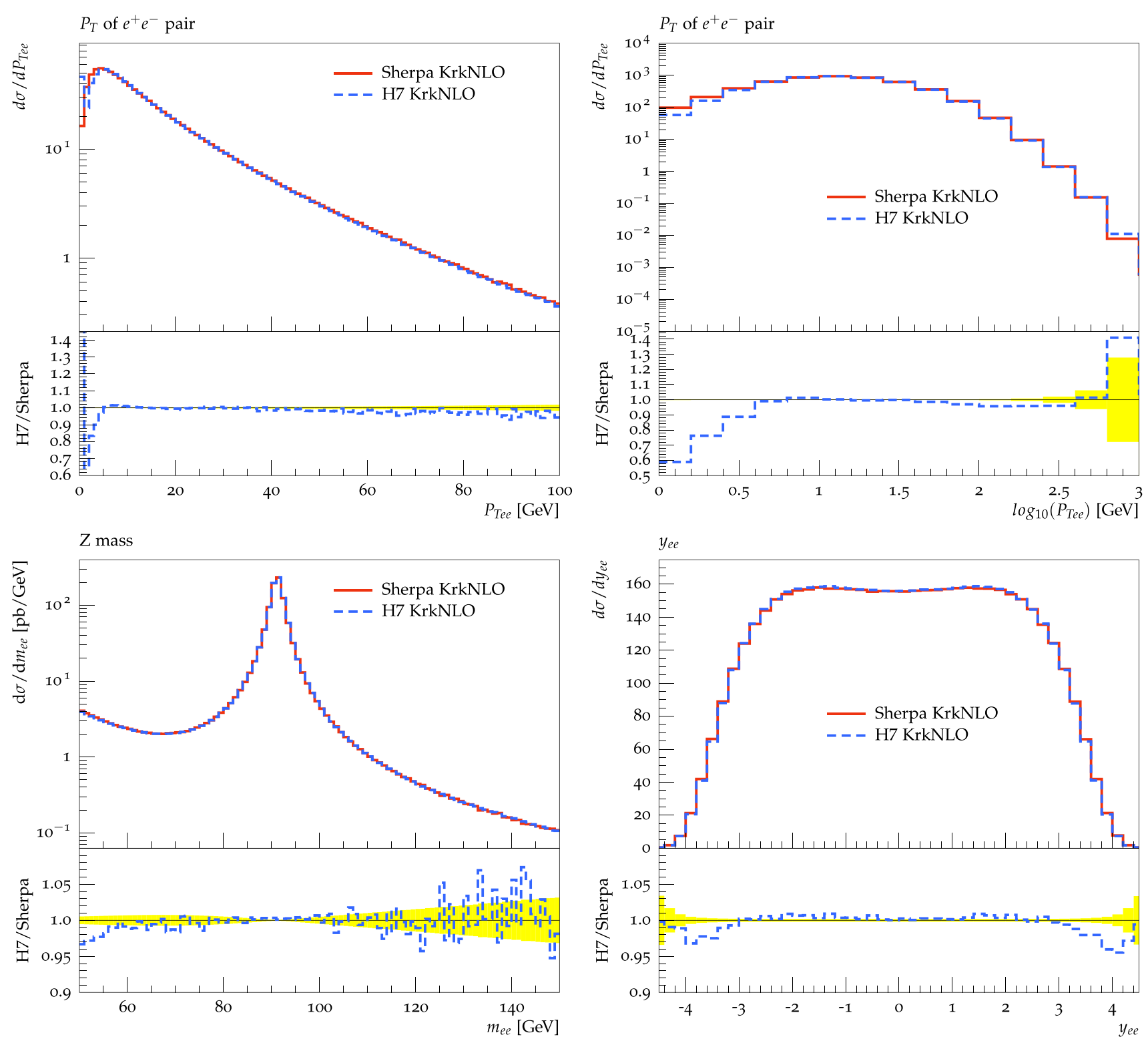

Fig. 2 Comparisons of the $Z / \gamma^{*}$ distributions from the KrkNLO method, as implemented in Sherpa and Herwig 7, for the Drell-Yan process with $e^{+} e^{-}$pairs in the final state; see text for details

In Fig. 2, we show similar distributions as in Fig. 1, but this time include the NLO QCD corrections according to the KrkNLO method. Again, a good agreement between the two programs is found. Only in the low $p_{T}$ region of the $p_{T e e}$ distributions are some differences visible, but they reflect effects already seen in Fig. 1. Given this agreement we are able to validate our implementation of the KrkNLO method in Herwig 7.

\subsubsection{KrkNLO results: $M C$ vs. $\mathbf{M C}_{\mathbf{D Y}}$ prescriptions}

Having validated the implementation of the KrkNLO method in Herwig 7 we are ready to present the first new results. We start from the comparison of the KrkNLO predictions based on the $\mathrm{MC}_{\mathrm{DY}}$ PDFs, defined in Ref. [25], with those in which the complete MC-scheme PDFs, first introduced in Ref. [34], are used. The main difference between the $\mathrm{MC}_{\mathrm{DY}}$ and MC PDFs is that in the former only the quark PDFs are transformed from the $\overline{\mathrm{MS}}$ to $\mathrm{MC}$ scheme and the gluon PDF is unchanged, whereas in the latter the gluon PDF is also transformed to the MC scheme; this is described in Ref. [34].

We note that for the DY process the transformation of the gluon PDF comes as an effect beyond NLO, so formally, for any predictions at the $\mathrm{NLO}$ level, the $\mathrm{MC}_{\mathrm{DY}} \mathrm{PDFs}$ are sufficient. However, this is not the case for processes in which initial-state gluons are present at the LO level, as is the case for Higgs-boson production in gluon-gluon fusion. 
Therefore, for future applications of the KrkNLO method to a generic process we shall use the complete MC PDFs of Ref. [34].

Table 3 Values of the total cross section with statistical errors for the Drell-Yan process from KrkNLO implemented in Herwig 7 for two variants of MC-scheme PDFs compared with the fixed-order NLO result from MCFM; see text for details

\begin{tabular}{llll}
\hline & MCFM: & KrkNLO: & KrkNLO: \\
& MS PDFs & $\begin{array}{l}\text { MCPY } \\
\text { PDFs }\end{array}$ & MC PDFs \\
\hline$\sigma_{\text {tot }[\mathrm{pb}]}$ & $1086.5(1)$ & $1046.5(2)$ & $1022.3(2)$ \\
\hline
\end{tabular}
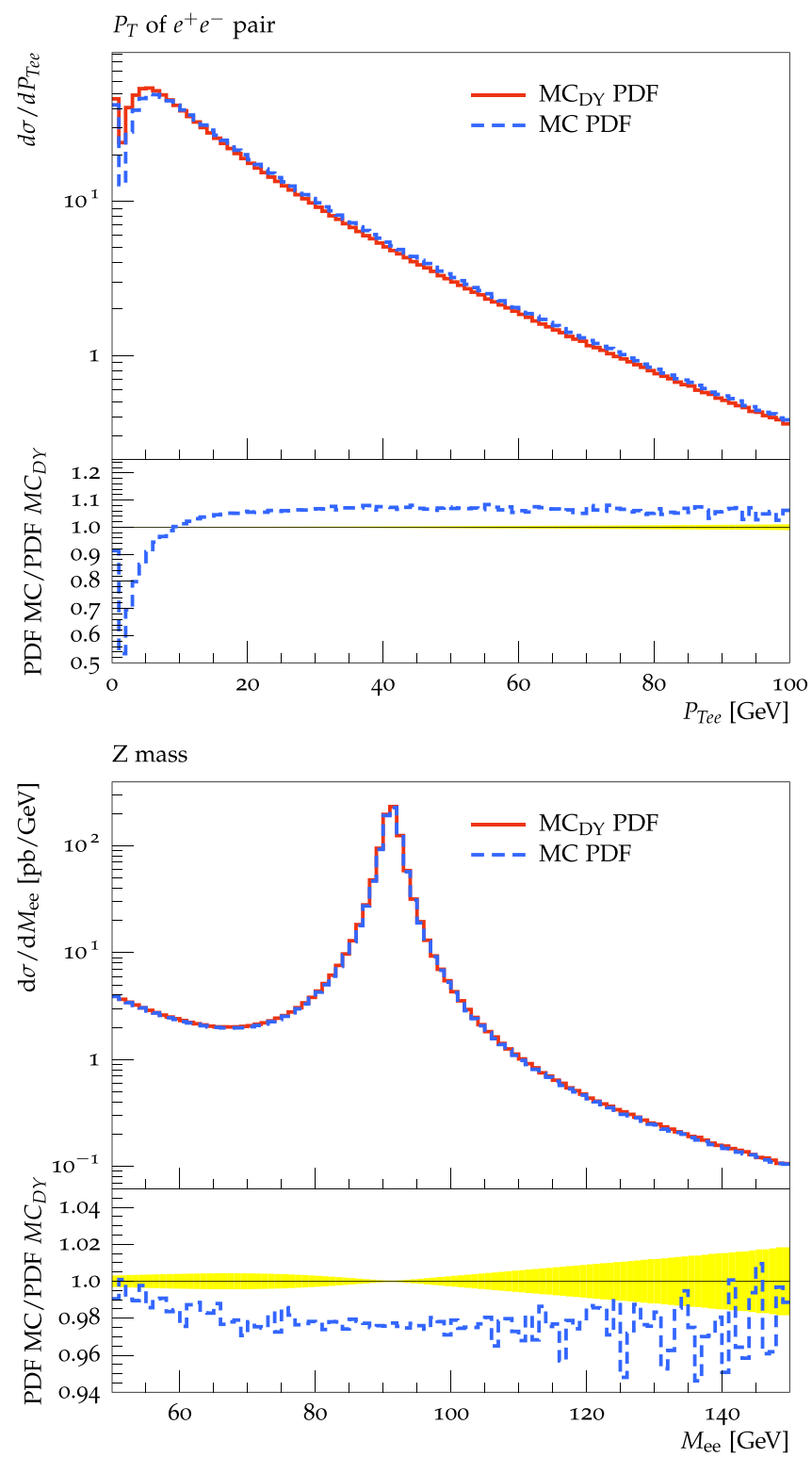

From Table 3 we see that the differences between the values of the total cross section corresponding to these two variants of the MC-scheme PDFs are rather small, $\sim 2 \%$, well within uncertainties of the NLO predictions. For comparison, we also show the fixed-order NLO result obtained from

Table 4 Values of the total cross section with statistical errors (in brackets) at the LO level for Higgs production in gluon-gluon fusion at the LHC for the $\overline{\mathrm{MS}}$ CT10nlo $\left(\alpha_{s}=0.118\right)$ PDFs from HNNLO and Herwig 7 (fixed order); see text for details

\begin{tabular}{lll}
\hline & HNNLO@LO & Herwig 7 \\
\hline$\sigma_{\text {tot }}[\mathrm{pb}]$ & $5.565(1)$ & $5.564(1)$ \\
\hline
\end{tabular}
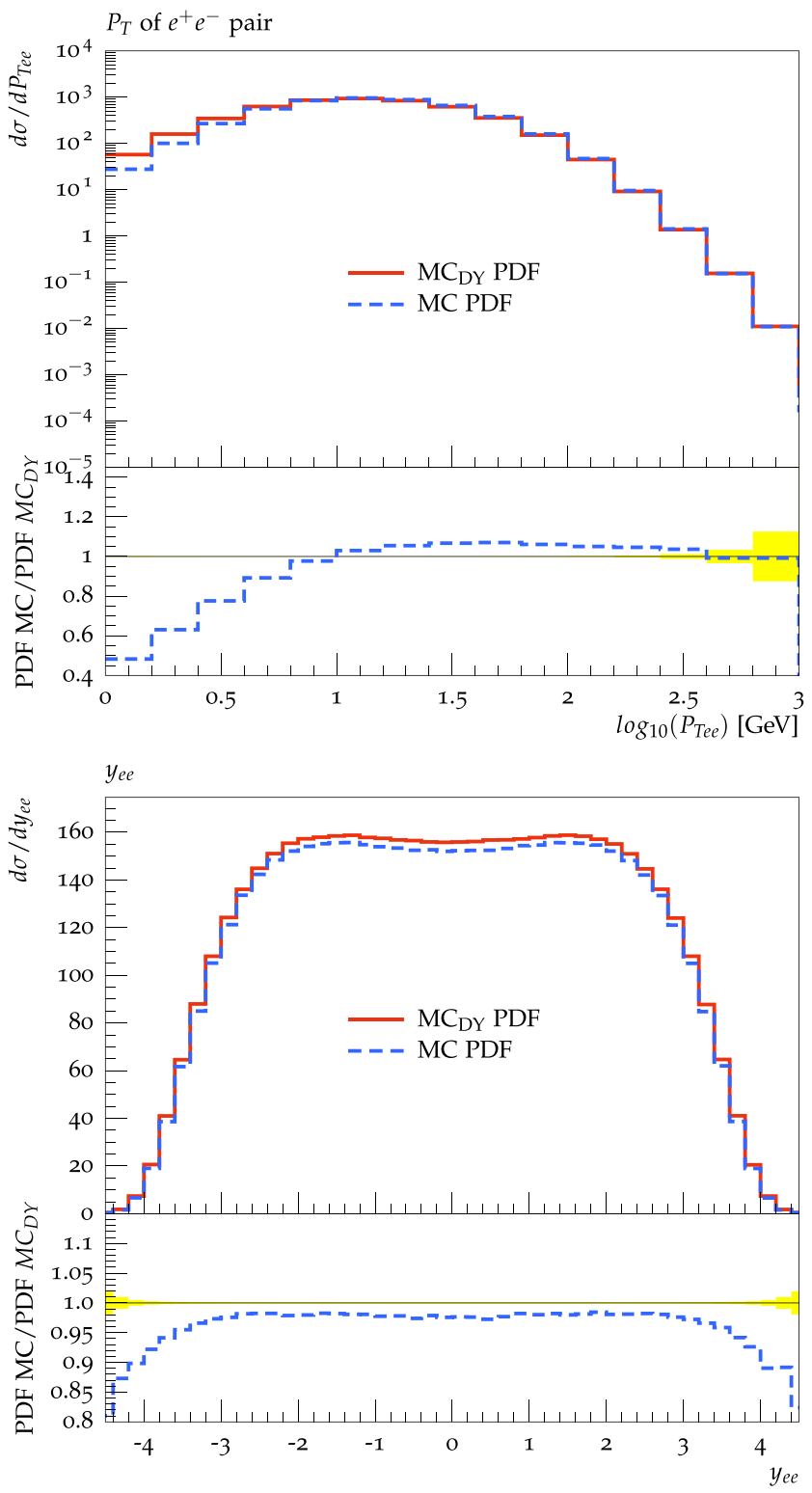

Fig. 3 Comparisons of the $Z / \gamma^{*}$ distributions, as in the previous plots, from KrkNLO implemented in Herwig 7 for both the MC $C_{D Y}$ PDFs and the complete MC PDFs. In the KrkNLO, the NLO weights use $\alpha_{s}\left(q^{2}\right)$ in the hard-real corrections and $\alpha_{s}\left(M_{Z}^{2}\right)$ in the virtual+soft-real ones 
Table 5 Values of the NLO total cross section with statistical errors (in brackets) for Higgs-boson production in gluon-gluon fusion at the LHC for KrkNLO, MC@NLO and POWHEG as calculated by Herwig 7, as well as the NLO and NNLO result from HNNLO; see text for details

\begin{tabular}{lllllll}
\hline & MC@NLO & POWHEG & & KrkNLO & & \multicolumn{2}{l}{ HNNLO } \\
\cline { 3 - 4 } & & Default & Original & & NLO & NNLO \\
\hline$\sigma_{\text {tot }[\mathrm{pb}]}$ & $12.700(2)$ & $12.699(2)$ & $12.697(2)$ & $12.939(2)$ & $12.640(1)$ & $17.063(15)$ \\
\hline
\end{tabular}

MCFM using the $\overline{\mathrm{MS}}$ PDFs. The differences with respect to the KrkNLO results are at the level of 4-6\%, also within the NLO accuracy. For the distributions of the quantities as shown in the previous plots, presented in Fig. 3, the differences are at the level of a few per-cent, except for in the low $p_{T}$ region where they can grow up to $\sim 50 \%$, but this region is very sensitive to soft gluon effects (and thus to the gluon PDF) that are formally beyond the NLO approximation.

\subsection{Higgs-boson production}

In this section we present results for Higgs-boson production in gluon-gluon fusion at the LHC obtained with the KrkNLO method implemented in Herwig 7 and compare them with predictions of other NLO-PSMC matching approaches, namely MC@NLO [30] and POWHEG [32] as implemented in Herwig 7, as well as with fixed-order calculations at NLO and NNLO from HNNLO [5] and an NNLL+NNLO calculation from HqT $[7,36]$. We opt to use the CT10n1。 PDF set [46] which has $\alpha_{s}\left(M_{Z}^{2}\right)=0.118$, and set Herwig 7 equivalently, such that we have a consistent $\alpha_{s}$ setting across all predictions. ${ }^{3}$

Finally, we confront the theoretical predictions of all the above matching methods with experimental measurements performed at the LHC during Run 1 by the ATLAS collaboration [37].

\subsubsection{LO results}

We start from the numerical cross-check at the LO level of different programs used in our study. In Table 4 we show the results for the total cross section obtained from HNNLO and Herwig 7 (fixed order). These values are in very good agreement, despite small differences in the running of $\alpha_{s}$. We are therefore ensured that all of the parameters as well as PDFs used in computation of Higgs-boson production in gluon-gluon fusion are consistently set in these programs.

\subsubsection{NLO results}

Here we present the results from KrkNLO alongside those of the MC@NLO and POWHEG methods implemented

\footnotetext{
3 Aside from small differences in the running $\alpha_{s}$ between HNNLO and Herwig 7.
}

Total cross section for Higgs production via gluon-fusion

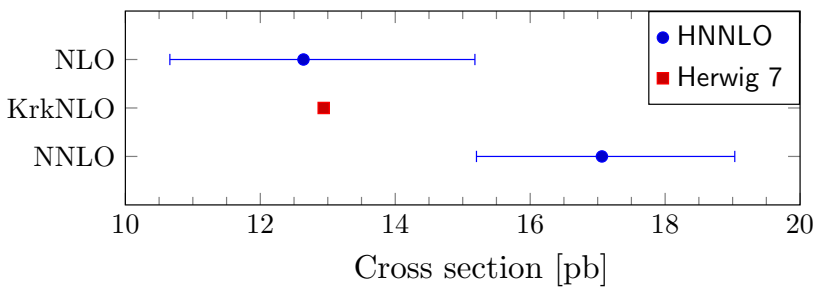

Fig. 4 Comparison of the total cross section for Higgs-boson production in gluon-gluon fusion at NLO, from HNNLO and KrkNLO, as well as at NNLO from HNNLO. The error bars, shown for HNNLO, are obtained from the independent variations of the renormalization and factorization scales by factors of $1 / 2$ and 2 from $M_{H}$

in Herwig 7 as well as the NLO and NNLO results from HNNLO as well as a results from HqT. The KrkNLO setup uses $\alpha_{s}\left(q^{2}\right)$ for the hard-real corrections and $\alpha_{s}\left(M_{H}^{2}\right)$ for the virtual+soft-real corrections. We show two variants of the POWHEG method: The first one, POWHEG (Default), is the default set-up in Herwig 7 and it restricts the transverse momentum of parton-shower emissions to be less than the factorization scale, as is done in the MC@NLO set-up, which follows the initial work of Ref. [29]; the second one, POWHEG (Original), is closer to its original implementation [32] which has no such restriction. This amounts to POWHEG (Default) generating both $\mathrm{S}$ and $\mathrm{H}$-events, with POWHEG (Original) only generating S-events.

The values of the total cross section, with statistical errors, for the Higgs-boson production process are shown in Table 5. We can see that, as expected, both the MC@NLO and the POWHEG results give the same total cross sections. The KrkNLO method gives a slightly higher value of the cross section than the other methods. This can be explained by the beyond-NLO contributions that are partially accounted for in the KrkNLO result. Additionally, in Fig. 4 we show the total cross sections from HNNLO, along with error bands generated by the variation of the renormalization and factorization scales by factors of $1 / 2$ and 2 around $M_{H}$. We see that the prediction of KrkNLO is within the NLO uncertainty estimate. Furthermore, we see that the uncertainty estimate for NNLO is still rather large, at around 10\%, and does not overlap with the NLO range.

In Fig. 5 we present the distributions of the Higgs-boson transverse momentum $p_{T}^{\mathrm{H}}$ and rapidity $y_{\mathrm{H}}$, comparing the results from KrkNLO with the ones from MC@NLO and 

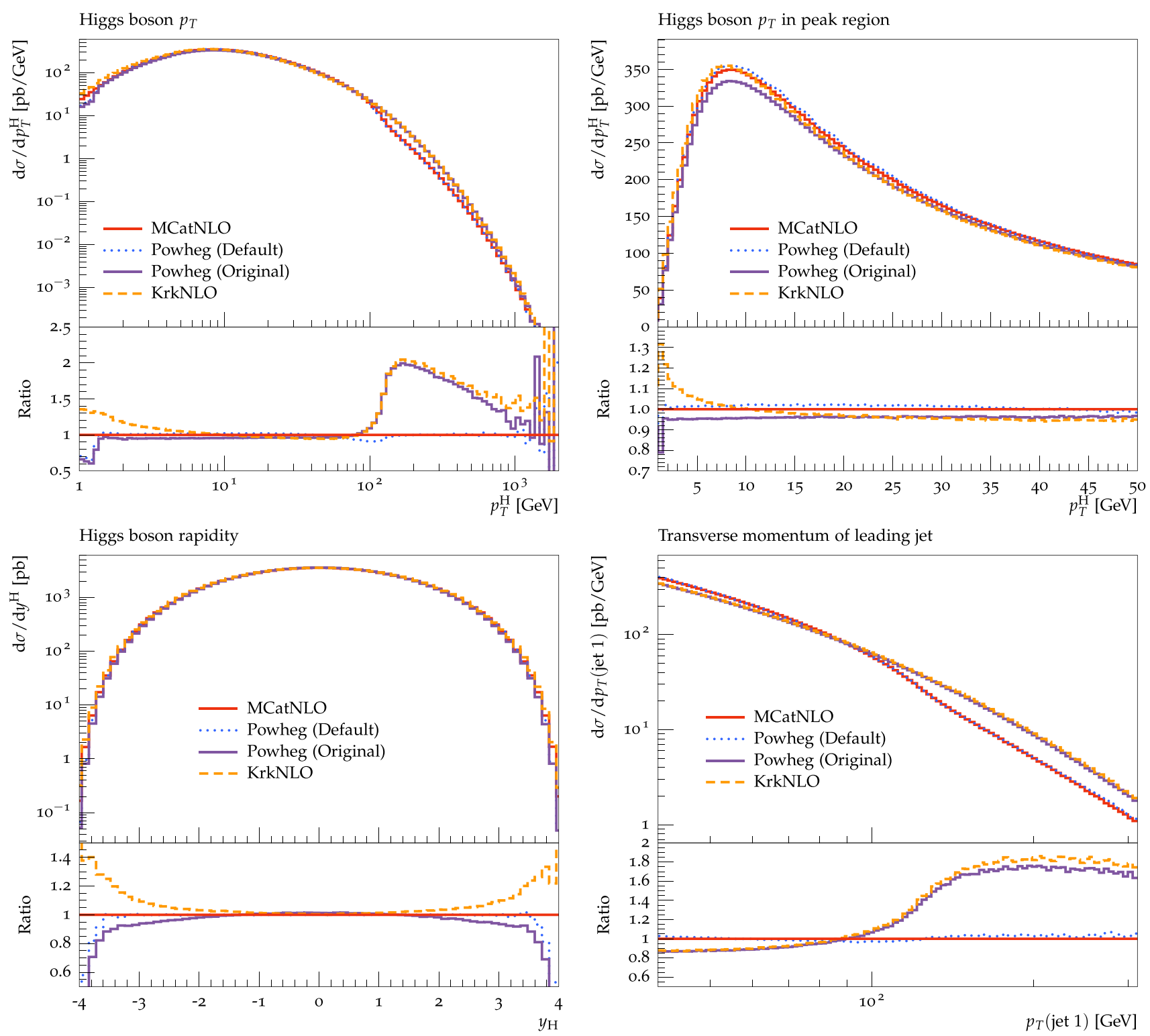

Fig. 5 Comparisons of the Higgs-boson transverse-momentum and rapidity distributions from the KrkNLO, MC@ NLO and POWHEG methods implemented in Herwig 7 for Higgs-boson production in gluon-gluon fusion at the LHC; see text for details

POWHEG. All of the predictions agree within a few per-cent for the $p_{T}^{\mathrm{H}}$ range from $\sim 5 \mathrm{GeV}$ to $\sim 100 \mathrm{GeV}$ and for the rapidity range $[-3,3]$. In the region $p_{T}^{\mathrm{H}}>M_{H}$ larger differences between KrkNLO and MC@NLO/POWHEG (Default) are visible, whereas KrkNLO produces a similar shape to POWHEG (Original). The main reason for this is that both MC@NLO and POWHEG (Default) restrict the value of the transverse momentum of parton-shower emissions to be below the value of $M_{H}$, whereas for KrkNLO, and also POWHEG (Original), there is no such restriction; this can be seen in the upper-left plot, where a sharp drop of the $p_{T}$ spectrum for $p_{T} \gtrsim M_{H}$ is visible in the case where the emissions are restricted by this hard-cutoff. However, this spectrum can be smoothed by relaxing this condition, as shown in Refs. [47,48]. Of course, such differences are acceptable within the NLO approximation.

Next, in Fig. 6 we compare KrkNLO, MC@NLO and POWHEG predictions from Herwig 7 for the Higgs-boson transverse-momentum distribution with the corresponding result obtained from HNNLO $[5,16]$ and $\mathrm{HqT}[7,36]$. The error bands HNNLO NNLO distributions were obtained by varying the renormalization and factorization scales by the typical factors of $1 / 2$ and 2 around $M_{H}$ as an estimate of the uncertainty from neglected higher orders. The HqT distributions were obtained similarly, but also include variations of the resummation scale of $1 / 2$ and 2 at the central value of $M_{H} / 2$ as recommended in Ref. [36]. The HNNLO comparison, upper plot, is shown relative to the NLO distribution 

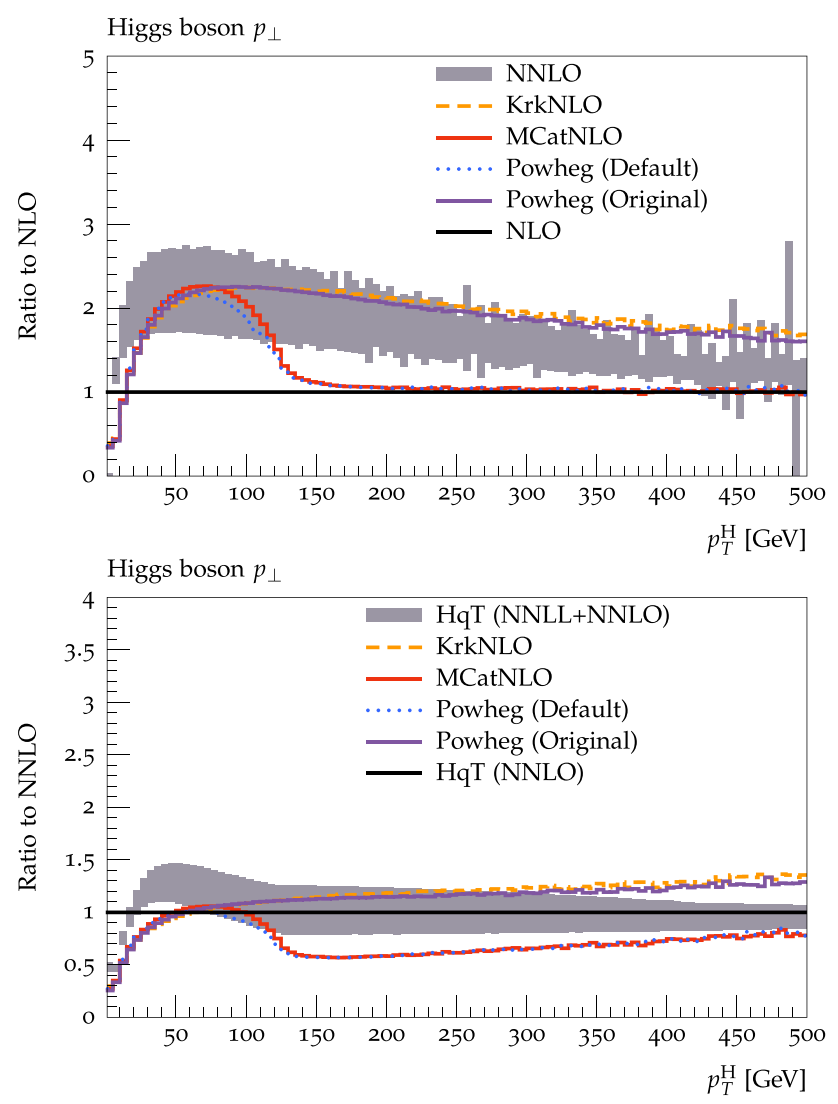

Fig. 6 Higgs-boson transverse-momentum distributions from KrkNLO, POWHEG and MC@NLO. The upper plot compares our results with the fixed-order NNLO result from the HNNLO program and are shown relative to the NLO results from HNNLO. The lower plot shows our results in comparison to $\mathrm{HqT}$, these are shown relative to the HqT NNLO prediction. The content of the error bands is described in the main text

from HNNLO and the HqT comparison, lower plot, is shown relative to the NNLO distribution from $\mathrm{HqT}$.

As we see in the upper plot of Fig. 6, both the KrkNLO and the NNLO results show the trends of quickly raising above the NLO result at low and moderate $p_{T}^{\mathrm{H}}$ and remaining above it at high $p_{T}^{\mathrm{H}}$. The fact that our method gives a result that is higher than the NLO one at high $p_{T}^{\mathrm{H}}$ is a consequence of the mixed real-virtual $\mathcal{O}\left(\alpha_{s}{ }^{2}\right)$ terms, which constitute part of the NNLO correction and arise because of the multiplicative nature of the KrkNLO approach.

In the upper plot of Fig. 6 we also show similar comparisons with NNLO for MC@NLO and two versions of POWHEG. The behaviour at low $p_{T}^{\mathrm{H}}$ is close to that from KrkNLO. At high $p_{T}^{\mathrm{H}}$, however, MC@NLO and POWHEG (Default), by construction, converge to the NLO results, departing from the NNLO predictions. On the other hand, KrkNLO and POWHEG (Original) are closer to the NNLO predictions but for larger $p_{T}^{\mathrm{H}}$ values they are marginally harder. The lower plot of Fig. 6 shows results from MC@NLO,POWHEG and KrkNLO compared to the resummed calculation from HqT (for the "switched" option).
All of the NLO+PS give similar results up to roughly $80 \mathrm{GeV}$. The HqT result peaks towards lower values of $p_{T}^{\mathrm{H}}$ compared to the other predictions.

In Fig. 7 we show the results of the KrkNLO method obtained for different modern $\overline{\mathrm{MS}}$ PDF sets: CT10nlo (as used in this section), CT1410, HERAPDF 2 0, MMHT2 01410 and NNPDF3010. Except for CT10, we have used the LO versions of the corresponding PDF parametrizations, since at NLO they become negative at some regions of the $x$ variable and this poses a problem for the Herwig 7 PSMC generator. We can see that the distributions can vary even by up to $40 \%$. The biggest differences are observed at low transverse momenta and large rapidities. In Appendix 1 we compare all the different PDFs in the $\overline{\mathrm{MS}}$ and MC schemes and show that the differences at the level of parton distribution functions; see Fig. 9, are commensurate to those observed in Fig. 7 for the differential cross sections. Further studies of systematic effects due to PDFs are left for the future.

Finally, in Fig. 8 we compare the predictions for the distributions of the Higgs-boson transverse momentum and rapidity, the number of jets and the transverse momentum of the hardest jet from KrkNLO, MC@NLO and POWHEG with the ATLAS data collected in LHC Run 1 with a collision energy of $\sqrt{s}=8 \mathrm{TeV}$ [37]. To our generated results for the gluon-gluon fusion we have added the contributions from other Higgs-production channels, denoted $\mathrm{XH}$, taken from Ref. [37] - they account for $\sim 12 \%$ of the cross section. All of the data points lie above the theoretical predictions, although the experimental errors are large, The NLOPSMC matching methods offer largely equivalent predictions with KrkNLO and POWHEG (Original) predicting slightly harder spectrum for high $p_{\perp}$ and higher rates for larger jet multiplicities (similar trends are also seen in Fig. 5). Further differences were previously discussed in the context of Fig. 5. In our simulations we have used the CT10nlo PDF parametrization, the same that was used in Ref. [37] for theoretical predictions. However, we have checked that changes of our results due to the use of different PDF sets, discussed in Appendix A, are much smaller than the experimental errors of the ATLAS data and negligible compared to the differences between these results and the data points.

\section{Conclusions and outlook}

In this paper we have presented the numerical results of the KrkNLO method for the Drell-Yan (DY) process and Higgsboson production in gluon-gluon fusion at the LHC for the collision energy $\sqrt{s}=8 \mathrm{TeV}$.

Firstly, we have validated the implementation of the KrkNLO method in the Herwig 7 PSMC by comparing its results for the DY process with previous results obtained with the Sherpa-based implementation. Then we have presented new results for the DY process with the complete MC-scheme 

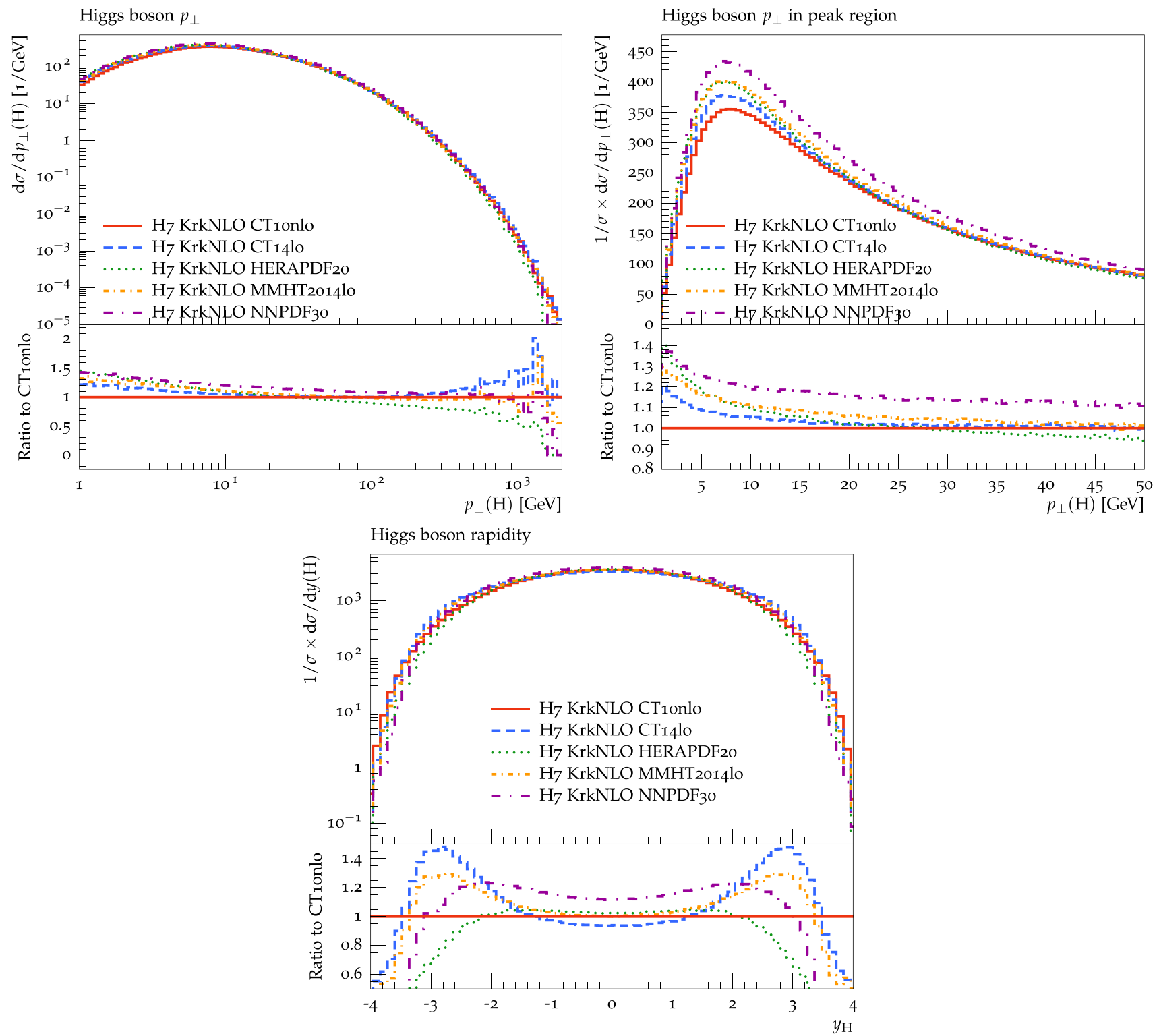

Fig. 7 Comparisons of the Higgs-boson transverse-momentum and rapidity distributions from the KrkNLO method using different PDF sets in the MC factorization scheme for the Higgs-boson production in gluon-gluon fusion at the LHC; see text for details

PDFs that were recently defined in Ref. [34]. These results have been compared with those for the older variant of the MC-scheme PDFs, called here the $\mathrm{MC}_{\mathrm{DY}} \mathrm{PDF}$, which were introduced in Ref. [25] exclusively for the DY process. The agreement between the results for these two variants of the MC-scheme PDFs has been found at the level of $\sim 2 \%$, which is well within the NLO accuracy.

Our main numerical results in this paper concern the Higgs-boson production process. We have presented the results for the total cross section, as well as distributions of the Higgs-boson transverse momentum and rapidity. The KrkNLO predictions have been compared with those of the MC@NLO and POWHEG methods. A good agreement, within the NLO accuracy, has been found between the default options of these methods. For $p_{T}^{\mathrm{H}}>M_{H}$ the KrkNLO result shows better agreement with the POWHEG (Original) option, a result of the restriction on the transverse momentum of the parton-shower emissions to below the factorization scale present in the other set-ups.

Finally, the theoretical predictions of the above NLOPSMC matching methods have been compared with the ATLAS data from LHC Run 1 for several observables for the Higgs-production process. All of the matching methods underestimate the ATLAS measurements, however, the experimental errors are large. The KrkNLO results offer comparable predictions to other matching methods in all dis- 

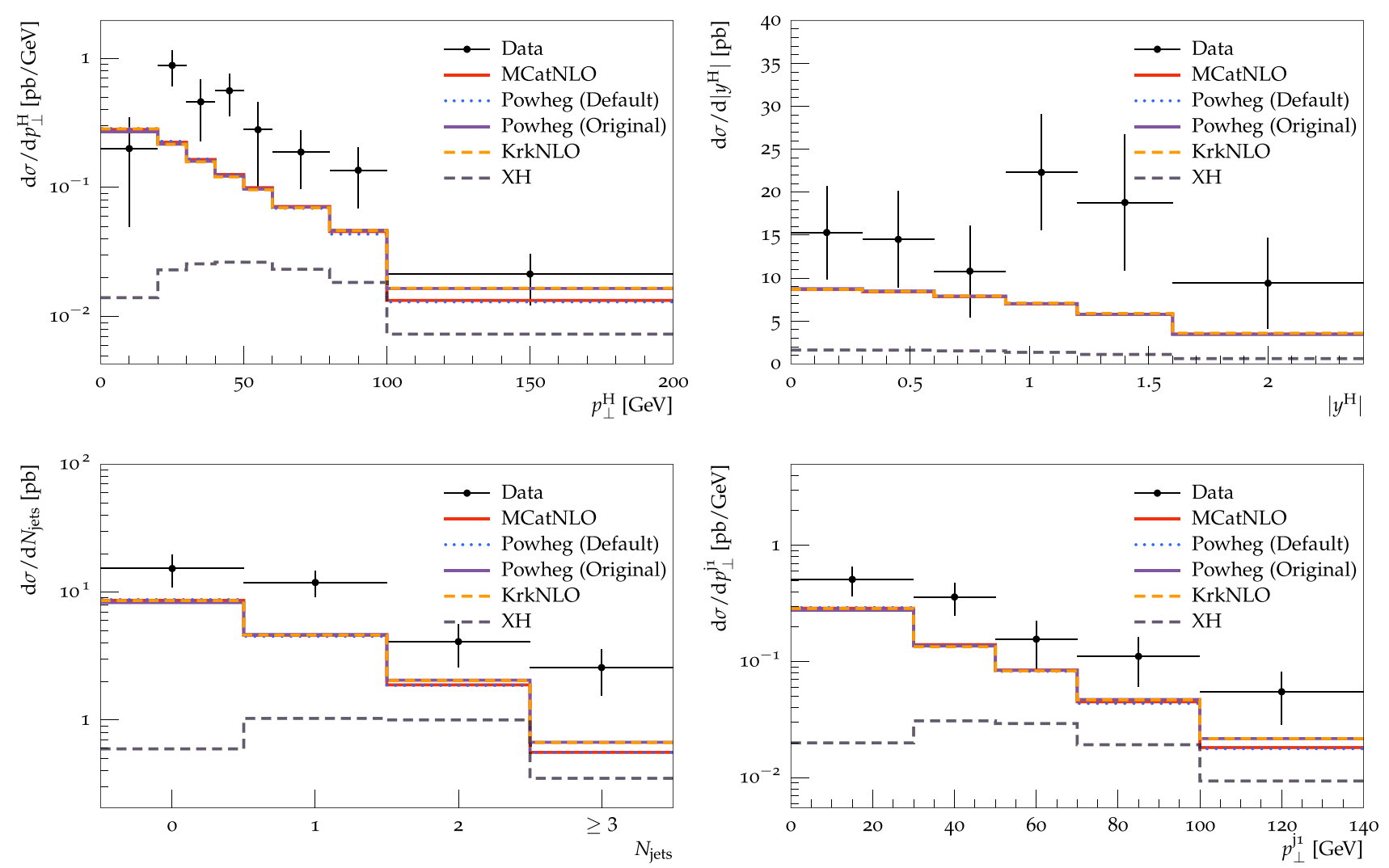

Fig. 8 Comparisons of the predictions of the KrkNLO, MC @ NLO and POWHEG methods implemented in Herwig 7 for Higgs-boson production with the ATLAS data from LHC Run 1. The gluon-gluon fusion results from Herwig 7 are plotted on top of the XH results from Ref. [37].

tributions and, along with POWHEG (Original), predict marginally harder spectrum for high $p_{T}$ and larger jet multiplicities. It is worth mentioning that all the calculations are performed in the infinite top-quark mass approximation, therefore including finite quark mass effects, which are important for large transverse momenta, would most likely bring the predictions closer to the experimental data.

As a next step in our numerical predictions with the KrkNLO method we plan to perform a more detailed study of the Drell-Yan processes, involving both the neutral $\left(Z / \gamma^{*}\right)$ and the charged $\left(W^{ \pm}\right)$modes, in the presence of experimental cuts and a focus on leptonic observables. In order to do this, we need to supplement the NLO-correcting weights with appropriate spin correlations for vector-boson decay products (which is not needed in the case of the scalar Higgs boson). This can be done rather easily within the Herwig 7 PSMC algorithm using the method proposed in Ref. [49]. Future work will seek to apply the KrkNLO method to other processes investigated at the LHC, first of all looking at electroweak-boson pair-production $(V V$, where $V=\gamma^{*}, Z, W^{+}, W^{-}$) and $V+$ jet production. This would be an important test of feasibility and universality of the method.

Future work will also comprise an appraisal of uncertainties of the KrkNLO approach, similar to that of Ref. [50]. Beyond this, the natural extension for KrkNLO is NNLO
+ NLOPS, where NLOPS is PSMC that implements the NLO evolution kernels in the fully exclusive form, and thus provides the full set of the soft-collinear counter-terms for the hard process. Reference [24] reviews several feasibility studies showing that construction of such a NLOPS is, in principle, plausible. Moreover, the simplifications due to the KrkNLO method in achieving NLO+PS will, in our opinion, be instrumental towards these more ambitions research directions.

The KrkNLO project will be available on hepforge at https://krknlo.hepforge.org. This will become the home site of the KrkNLO development, containing relevant codes and the MC-scheme PDFs as well as set-up instructions to facilitate its use within Herwig 7.

Acknowledgements We are grateful to Simon Plätzer for the useful discussions and his help with the dipole parton shower implemented in Herwig 7. We are indebted to the Cloud Computing for Science and Economy Project (CC1) at IFJ PAN (POIG 02.03.03-00-033/0904) in Kraków whose resources were used to carry out all the numerical computations for this project. We also thank Mariusz Witek and Miłosz Zdybał for their help with CC1. This work was funded in part by the MCnetITN FP7 Marie Curie Initial Training Network PITN-GA-2012315877.

Open Access This article is distributed under the terms of the Creative Commons Attribution 4.0 International License (http://creativecomm 
ons.org/licenses/by/4.0/), which permits unrestricted use, distribution, and reproduction in any medium, provided you give appropriate credit to the original author(s) and the source, provide a link to the Creative Commons license, and indicate if changes were made.

Funded by SCOAP ${ }^{3}$.

\section{Appendix: PDFs in $\overline{\mathrm{MS}}$ and MC schemes}

In this appendix we present comparisons of parton the distribution functions used in our study. Figure 9 shows the $\overline{\mathrm{MS}}$ and MC PDFs for the gluon and for the $u$ quark (remaining quark flavours look very similar). The MC PDFs were obtained from the $\overline{\mathrm{MS}}$ sets using the convolutions discussed in Ref. [34].

We observe that the gluon PDF in the MC scheme is larger at small and moderate $x$ than the $\overline{\mathrm{MS}} \mathrm{PDF}$. On the contrary, the quark PDFs are smaller in the MC scheme. We also see that various $\overline{\mathrm{MS}}$ sets exhibit differences that carry on to the MC scheme. These differences lead to a range of predictions shown in Fig. 7.
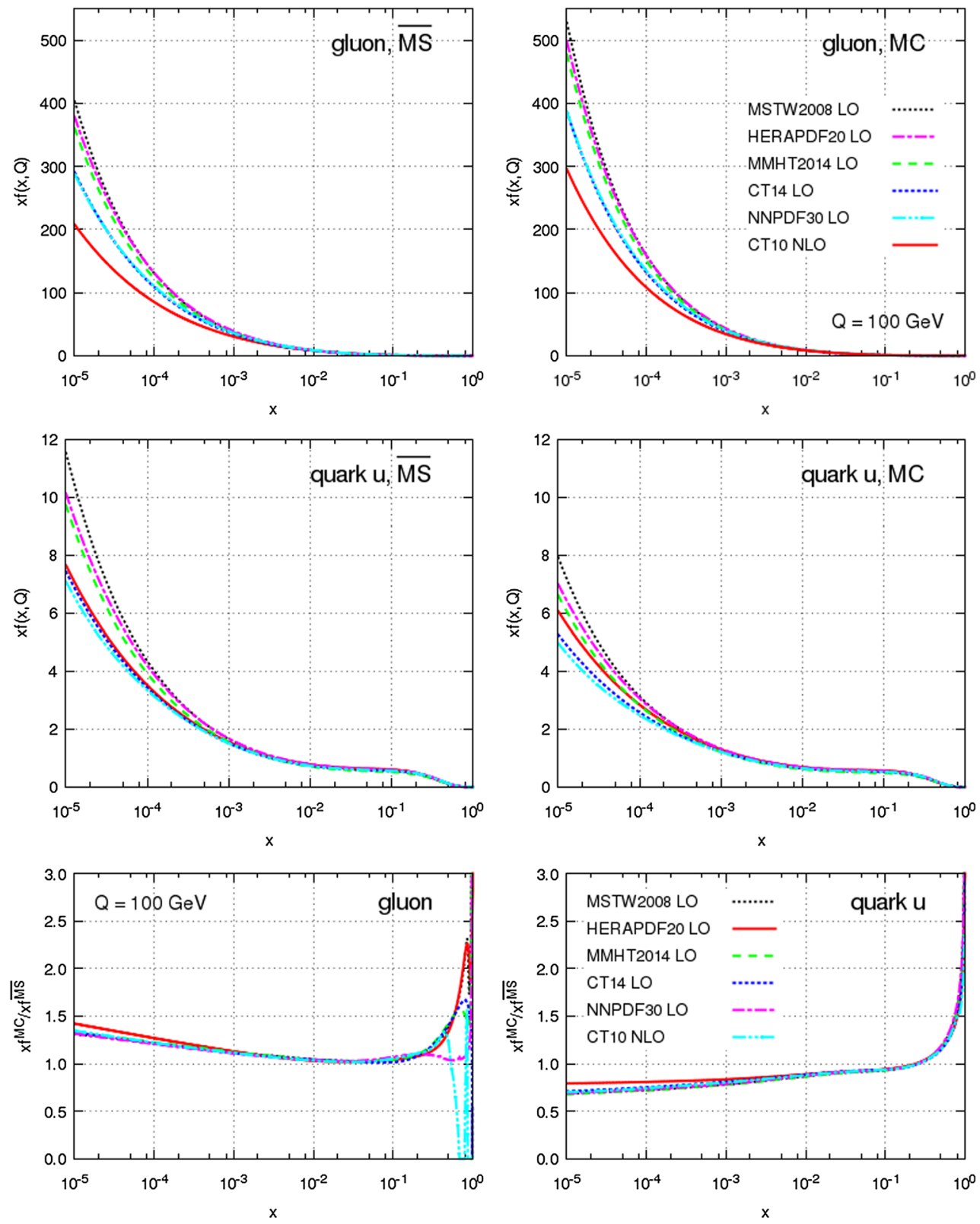

Fig. 9 Comparison of different sets of parton distributions functions in the $\overline{\mathrm{MS}}$ and MC factorization schemes 


\section{References}

1. ATLAS Collaboration, G. Aad et al., Phys. Lett. B 716, 1-29 (2012). arXiv:1207.7214

2. CMS Collaboration, S. Chatrchyan et al., Phys. Lett. B 716, 30-61 (2012). arXiv: 1207.7235

3. R.V. Harlander, W.B. Kilgore, Phys. Rev. Lett. 88, 201801 (2002). arXiv:hep-ph/0201206

4. C. Anastasiou, K. Melnikov, Nucl. Phys. B 646, 220-256 (2002). arXiv:hep-ph/0207004

5. S. Catani, M. Grazzini, Phys. Rev. Lett. 98, 222002 (2007). arXiv:hep-ph/0703012

6. C. Anastasiou, C. Duhr, F. Dulat, F. Herzog, B. Mistlberger, Phys. Rev. Lett. 114, 212001 (2015). arXiv: 1503.06056

7. D. de Florian, G. Ferrera, M. Grazzini, D. Tommasini, JHEP 11, 064 (2011). arXiv:1109.2109

8. P.F. Monni, E. Re, P. Torrielli, Phys. Rev. Lett. 116(24), 242001 (2016). arXiv:1604.02191

9. T. Becher, M. Neubert, D. Wilhelm, JHEP 05, 110 (2013). arXiv:1212.2621

10. K. Hamilton, P. Nason, G. Zanderighi, JHEP 1210, 155 (2012). arXiv: 1206.3572

11. K. Hamilton, P. Nason, C. Oleari, G. Zanderighi, JHEP 1305, 082 (2013). arXiv:1212.4504

12. S. Hoeche, Y. Li, S. Prestel, Phys. Rev. D 90(5), 054011 (2014). arXiv: 1407.3773

13. K. Hamilton, P. Nason, E. Re, G. Zanderighi, JHEP 10, 222 (2013). arXiv: 1309.0017

14. R. Frederix, K. Hamilton, JHEP 05, 042 (2016). arXiv: 1512.02663

15. K. Hamilton, T. Melia, P.F. Monni, E. Re, G. Zanderighi, JHEP 09, 057 (2016). arXiv:1606.07062

16. M. Grazzini, JHEP 02, 043 (2008). arXiv:0801.3232

17. S. Hoeche, Y. Li, S. Prestel, arXiv: 1405.3607

18. S. Alioli, C.W. Bauer, C. Berggren, F.J. Tackmann, J.R. Walsh et al., JHEP 1406, 089 (2014). arXiv: 1311.0286

19. S. Hoeche, S. Prestel, Eur. Phys. J. C 75(9), 461 (2015). arXiv: 1506.05057

20. S. Alioli, C.W. Bauer, C. Berggren, F.J. Tackmann, J.R. Walsh, Phys. Rev. D 92(9), 094020 (2015). arXiv:1508.01475

21. K. Hamilton, P. Nason, G. Zanderighi, JHEP 05, 140 (2015). arXiv: 1501.04637

22. S. Jadach, A. Kusina, W. Płaczek, M. Skrzypek, M. Slawinska, Phys. Rev. D 87, 034029 (2013). arXiv:1103.5015

23. S. Jadach, W. Płaczek, S. Sapeta, A. Siódmok, M. Skrzypek, Acta Phys. Polon. B 46(11), 2089 (2015)

24. S. Jadach, A. Kusina, W. Płaczek, M. Skrzypek, Acta Phys. Polon. B 44(11), 2179-2187 (2013). arXiv: 1310.6090

25. S. Jadach, W. Płaczek, S. Sapeta, A. Siodmok, M. Skrzypek, JHEP 10, 052 (2015). arXiv: 1503.06849
26. S. Jadach, M. Jeżabek, A. Kusina, W. Płaczek, M. Skrzypek, Acta Phys. Polon. B 43, 2067 (2012). arXiv:1209.4291

27. T. Gleisberg et al., JHEP 02, 007 (2009). arXiv:0811.4622

28. J.M. Campbell, R.K. Ellis, Phys.Rev. D 60, 113006 (1999). http:// mcfm.fnal.gov. arXiv:hep-ph/9905386

29. S. Frixione, B.R. Webber, JHEP 06, 029 (2002). arXiv:hep-ph/0204244

30. S. Frixione, B.R. Webber, arXiv:hep-ph/0601192

31. P. Nason, JHEP 11, 040 (2004). arXiv:hep-ph/0409146

32. S. Frixione, P. Nason, C. Oleari, JHEP 0711, 070 (2007). arXiv:0709.2092

33. S. Catani, L. Cieri, G. Ferrera, D. de Florian, M. Grazzini, Phys. Rev. Lett. 103, 082001 (2009). arXiv:0903.2120

34. S. Jadach, W. Płaczek, S. Sapeta, A. Siodmok, M. Skrzypek, arXiv: 1606.00355

35. S. Jadach, W. Płaczek, S. Sapeta, A.K. Siodmok, M. Skrzypek, PoS LL2016, 020 (2016). arXiv:1607.00919

36. G. Bozzi, S. Catani, D. de Florian, M. Grazzini, Nucl. Phys. B 737, 73-120 (2006). arXiv:hep-ph/0508068

37. ATLAS Collaboration, G. Aad et al., Phys. Rev. Lett. 115(9), 091801 (2015). arXiv:1504.05833

38. G. Altarelli, M.L. Mangano, Proccedings of the workshop on standard model physics (and more) at the LHC. Yellow Report, CERN, 2000-04, 9 (2000)

39. M.R. Whalley, D. Bourilkov, R.C. Group, arXiv:hep-ph/0508110

40. A.D. Martin, W.J. Stirling, R.S. Thorne, G. Watt, Eur. Phys. J. C 63, 189-285 (2009). arXiv:0901.0002

41. M. Bahr, S. Gieseke, M. Gigg, D. Grellscheid, K. Hamilton et al., Eur. Phys. J. C 58, 639-707 (2008). arXiv:0803.0883

42. J. Bellm et al., Eur. Phys. J. C 76(4), 196 (2016). arXiv: 1512.01178

43. S. Catani, M.H. Seymour, Nucl. Phys. B 485, 291-419 (1997). arXiv:hep-ph/9605323

44. S. Schumann, F. Krauss, JHEP 0803, 038 (2008). arXiv:0709.1027

45. S. Platzer, S. Gieseke, Eur. Phys. J. C 72, 2187 (2012). arXiv: 1109.6256

46. H.-L. Lai, M. Guzzi, J. Huston, Z. Li, P.M. Nadolsky, J. Pumplin, C.P. Yuan, Phys. Rev. D 82, 074024 (2010). arXiv:1007.2241

47. J. Alwall, R. Frederix, S. Frixione, V. Hirschi, F. Maltoni, O. Mattelaer, H.S. Shao, T. Stelzer, P. Torrielli, M. Zaro, JHEP 07, 079 (2014). arXiv:1405.0301

48. J. Bellm, G. Nail, S. Platzer, P. Schichtel, A. Siódmok, arXiv: 1605.01338

49. M.H. Seymour, Nucl. Phys. B 436, 443-460 (1995). arXiv:hep-ph/9410244

50. J. Bellm, S. Platzer, P. Richardson, A. Siódmok, S. Webster, Phys. Rev. D 94(3), 034028 (2016). arXiv:1605.08256 